\title{
GCU
}

Glasgow Caledonian

University

University for the Common Good

\section{Cytosolic lipid trafficking proteins STARD4 and STARD5 modulate hepatic neutral lipid metabolism: implications for diabetic dyslipidaemia and steatosis}

Soffientini, Ugo; Dolan, Sharron; Graham, Annette

Published in:

Journal of Diabetes and Metabolism

DOI:

$10.4172 / 2155-6156.1000558$

Publication date:

2015

Document Version

Publisher's PDF, also known as Version of record

Link to publication in ResearchOnline

Citation for published version (Harvard):

Soffientini, U, Dolan, S \& Graham, A 2015, 'Cytosolic lipid trafficking proteins STARD4 and STARD5 modulate hepatic neutral lipid metabolism: implications for diabetic dyslipidaemia and steatosis', Journal of Diabetes and Metabolism, vol. 6, no. 6, 1000558. https://doi.org/10.4172/2155-6156.1000558

\section{General rights}

Copyright and moral rights for the publications made accessible in the public portal are retained by the authors and/or other copyright owners and it is a condition of accessing publications that users recognise and abide by the legal requirements associated with these rights.

Take down policy

If you believe that this document breaches copyright please view our takedown policy at https://edshare.gcu.ac.uk/id/eprint/5179 for details of how to contact us. 


\title{
Cytosolic Lipid Trafficking Proteins STARD4 and STARD5 Modulate Hepatic Neutral Lipid Metabolism: Implications for Diabetic Dyslipidaemia and Steatosis
}

\author{
Ugo Soffientini, Sharron Dolan and Annette Graham*
}

Diabetes Research Group, Institute for Applied Health Research and the Department of Life Sciences, School of Health and Life Sciences, Glasgow Caledonian University, Glasgow, UK

\begin{abstract}
This study explored expression of the START domain family in hyperinsulinaemic, genetically obese rodents, and the functional roles of cytosolic StarD4 and StarD5 proteins in hepatic lipid synthesis and export. Genetic obesity in (fa/fa) Zucker rats repressed StarD4, StarD5, StarD8 and StarD13, and decreased levels of cytosolic StarD4 and StarD5 proteins, compared with lean controls, suggesting links with hepatic storage or secretion of lipids. Stable overexpression of STARD4 in rat McArdle RH7777 hepatoma cells increased lipidation of exogenous ApoA-I compared with empty vector (EV) control, modestly increased secretion of endogenously synthesized cholesterol and reduced incorporation of $\left[{ }^{14} \mathrm{C}\right]$ oleate into cellular cholesteryl esters. Synthesis and secretion of triacylglycerol did not change in STARD4 overexpressing cells. By contrast, STARD5 overexpression did not alter cholesterol synthesis, secretion, or lipidation of apoA-I, but increased synthesis of triacylglycerol from $\left[{ }^{3} \mathrm{H}\right]$ glycerol under basal conditions, enhanced incorporation of [ ${ }^{14} \mathrm{C}$ ]oleate into triacylglycerol, and increased expression of Dgat1 and Dgat2 compared with EV.
\end{abstract}

In summary, cytosolic STARD4 impacts predominantly on cholesterol efflux to apoA-I, implying directional transport of cholesterol to the plasma membrane, while STARD5 increases basal triacylglycerol synthesis and enriches this pool with exogenous fatty acids. Cytosolic StarD proteins may be involved in diabetic dyslipidaemia and steatosis.

Keywords: Insulin resistance; Lipid metabolism; High density lipoprotein; Steatosis; Dyslipidaemia; Cholesterol trafficking proteins

\section{Introduction}

Non-vesicular trafficking of lipids is mediated by two major gene families: the oxysterol-binding protein (OSBP) related proteins (ORPs) $[1,2]$ and the steroidogenic acute regulatory protein (StAR)-related lipid transfer (START) domain family [3-5]. The START domain is a $\sim 210$ amino acid $\alpha / \beta$ helix-grip fold, found in 15 distinct mammalian proteins (STARD1-D15), providing an adaptable hydrophobic lipid binding or sensing site for cholesterol, oxysterols, phospholipids, ceramides and small drug molecules. Phylogenetic analysis divides the family into six subfamilies, based on sequence and ligand similarities, and, more recently, comparative structural analysis [3-6]. In brief, the STARD1 (STARD1/D3) and STARD4 (D4/D5/D6) groups bind cholesterol and oxysterols, while the STARD2 (STARD2/D7/D10/ D11) group exhibits specificity for phospholipids and ceramides. Other members of the family include multi-domain proteins, linking the START domain with Rho GTPase (STARD8/D12/D13) or thioesterase (StARD14/D15) activities; the last member of the family is STARD9 [3-6].

Loss of function mutations in humans and genetic deletion studies in mice have revealed a range of phenotypes resulting from loss of differing members of the START domain family. Mutations affecting the function of the mitochondrial cholesterol trafficking protein, STARD1, cause congenital lipoid hyperplasia [7], while genetic deletion of StarD11 (CERT) which transports ceramides from the ER to the Golgi, is embryonic lethal in mice, due to mitochondrial disruption [8]. Targeted deletion of the RhoGAP START, Stard12, thought to be involved in regulating the formation of focal adhesion, also results in embryonic lethality, presumably due to loss of cytoskeletal organisation [9].
By contrast, deletion of the START domain from endosomal cholesterol transporter StarD3 results in modest changes in lipid metabolism [10]. Deletion of StarD4 leads to modest weight reduction, and decreased bile cholesterol and phospholipid concentrations in female mice; when challenged with a $0.5 \%$ cholesterol diet, female StarD4 (-/-) mice also evidenced moderate decreases in total cholesterol, LDL and cholesteryl ester concentrations [11]. Stard2/PCTP knockout mice exhibit a normal phenotype, but recent data suggest StarD2 as an important determinant of hepatic insulin sensitivity [12]. Finally, loss of Stard13, results in healthy, fertile mice, indicating that this protein cannot compensate for the loss of Stard12 (above) [13].

These studies have highlighted the differing roles of START family members in lipid sensing, trafficking and signalling, but relatively few studies have identified differences in expression of this family of proteins in disease states, such as obesity or diabetes, which are closely associated with cardiovascular disease. This study examined the expression of StarD lipid trafficking proteins in the Zucker ( $\mathrm{fa}$ / fa) rodent model of leptin receptor deficiency [14], and explored the

*Corresponding author: Annette Graham, Charles Oakley Laboratories Department of Life Sciences, School of Health and Life Sciences, 70 Cowcaddens Road, Glasgow G4 0BA, UK, Tel: +44(0) 141331 3722; Fax: +44(0) 1413313208 ; E-mail: Ann.Graham@gcu.ac.uk

Received April 15, 2015; Accepted May 28, 2015; Published May 30, 2015

Citation: Soffientini U, Dolan S, Graham A (2015) Cytosolic Lipid Trafficking Proteins STARD4 and STARD5 Modulate Hepatic Neutral Lipid Metabolism: Implications for Diabetic Dyslipidaemia and Steatosis. J Diabetes Metab 6: 558. doi:10.4172/2155-6156.1000558

Copyright: @ 2015 Soffientini U, et al. This is an open-access article distributed under the terms of the Creative Commons Attribution License, which permits unrestricted use, distribution, and reproduction in any medium, provided the original author and source are credited. 
Citation: Soffientini U, Dolan S, Graham A (2015) Cytosolic Lipid Trafficking Proteins STARD4 and STARD5 Modulate Hepatic Neutral Lipid Metabolism: Implications for Diabetic Dyslipidaemia and Steatosis. J Diabetes Metab 6: 558. doi:10.4172/2155-6156.1000558

functional role of cytosolic StarD4 and StarD5 proteins in hepatic synthesis and export of lipids by genetically manipulating expression of these proteins in rat McArdle RH7777 hepatoma cells.

\section{Methods}

Heterozygous Zucker rats $(\mathrm{Fa} / \mathrm{fa})$ were purchased from Harlan Laboratories, and sodium pentobarbital from J.M. Loveridge plc (Southampton). Tissue culture reagents were supplied by Lonza, and sterile tissue culture plastics from Greiner; STARD4 and STARD5 clones (pCMV) were purchased from Origene.com via Cambridge Biosciences. Apolipoprotein A-I was from Athens Research and Technology (Georgia, USA) and radiochemicals purchased from Perkin Elmer. Tri-Fast was from PeqLab, cDNA synthesis kits from Bioline, primers and probes (FAM/TAMRA) from EuroGenTech. Rabbit polyclonal antibodies to Gapdh, StarD4 and StarD5 were purchased from AbCAM, Santa Cruz and Biorbyt, respectively. Complete ${ }^{\mathrm{mm}}$ protease inhibitor cocktail was purchased from Roche. Chemicals, t.l.c.plates and solvents were purchased from Sigma Aldrich.

\section{Experimental animals}

Heterozygous Zucker rats $(\mathrm{Fa} / \mathrm{fa})$ were maintained in group $(n=3)$ cages on sawdust bedding, on a $14 \mathrm{~h}$-light/10h-dark cycle with standard chow and water provided ad libitum. Male lean $(\mathrm{Fa} / ? ; n=10)$ and obese $(f a / f a ; n=6)$, and female lean $(n=5)$ and obese $(n=4)$ rats were terminally anaesthetized at four months, using pentobarbital sodium $\left(100 \mathrm{mg} \mathrm{kg}^{-1}\right.$, I.P) [14]. Principles of laboratory animal care (NIHA publication no. 85-23, revised 1985 (http://grants1.nih.gov/grants/olaw/references/ phspol.htm) were followed. The study was approved by the institution's Animal Ethics and Welfare Committee, and procedures performed according to the UK Animals (Scientific Procedures) Act, 1986.

\section{Preparation of tissue samples}

Samples $(100 \mathrm{mg})$ of liver were suspended in Dulbecco's phosphate buffered saline ( $1 \mathrm{ml}$ ), using a Beadbeater (Thistle Scientific, UK), and a Bligh and Dyer (1959) lipid extraction performed [15]. Lipid extracts were dried under $\mathrm{N}_{2}$, and lipid mass measured, as described [16-18]. Total RNA from liver $(60 \mathrm{mg})$ was isolated after homogenisation in 500 $\mu \mathrm{l}$ of TriFast ${ }^{\mathrm{Tm}}$, genomic DNA removed by treatment with DNase, and cDNA prepared by reverse transcription; negative controls, omitting reverse transcriptase enzyme, were included for each sample. Hepatic protein lysates were prepared in RIPA buffer plus Complete ${ }^{\text {th }}$ protease inhibitor cocktail.

\section{Cell culture and experiments}

Rat McArdle RH7777 (CRL-1601; ATCC) cells, derived from female Rattus norvegicus (Morris hepatoma 7777) were maintained in DMEM (Dulbecco's Modified Eagle Medium) containing foetal bovine serum $(10 \% \mathrm{v} / \mathrm{v})$, penicillin/streptomycin $(50 \mathrm{U} / \mathrm{ml}, 50 \mu \mathrm{g} / \mathrm{ml}$ respectively), sodium bicarbonate $\left(\mathrm{NaCO}_{3} 0.06 \% \mathrm{w} / \mathrm{v}\right)$, HEPES buffer $(10 \mathrm{mM})$ and L-glutamine $(2 \mathrm{mM})$, at $37^{\circ} \mathrm{C}$ in a humidified atmosphere of $95 \%$ air and $5 \% \mathrm{CO}_{2}$. Transfection of McArdle cells with $2 \mu \mathrm{g}$ of empty vector (EV; pCMV.Neo) or this vector encoding full length human STARD4 or STARD5 was achieved using Amaxa NucleofectorII (Kit V, protocol T-028). Stable populations were selected using G418 (1.6 mg ml$\left.{ }^{-1}\right)$ and then maintained at $400 \mu \mathrm{g} \mathrm{ml}^{-1}$. Differences in nutrient partitioning between McArdle hepatoma cells and primary rat hepatocytes are described in [19].

Lipidation of apoA-I $\left(10 \mu \mathrm{g} \mathrm{ml}^{-1} ; 24 \mathrm{~h}\right)$ was assessed in cells labelled with $0.5 \mu \mathrm{Ci} \mathrm{ml}^{-1}\left[{ }^{3} \mathrm{H}\right]$ cholesterol, as described [16-18,20]. For lipid synthesis and secretion experiments, cells were cultured in serum-free
DMEM in presence of $\left[1-2{ }^{14} \mathrm{C}\right]$ acetate $(1.5 \mu \mathrm{Ci} / \mathrm{ml} ; 0.5 \mathrm{mM}),\left[1-{ }^{14} \mathrm{C}\right]$ oleate $(1 \mu \mathrm{Ci} / \mathrm{ml} ; 0.7 \mathrm{mM}$ complexed $1: 1$ with $\mathrm{BSA})$ or $\left[2-{ }^{3} \mathrm{H}\right]$ glycerol $(1.18 \mu \mathrm{Ci} / \mathrm{ml} ; 0.25 \mathrm{mM})$ for $2 \mathrm{~h}$, in the presence or absence of $0.35 \mathrm{mM}$ oleate/BSA (1:1) complex; concentrations of acetate $(0.5 \mathrm{mM})$ and glycerol $(0.25 \mathrm{mM})$ remained the same under all conditions tested. Note that the data for the EV control was previously reported in [20].

\section{Lipid analyses}

Samples of media were extracted according to Bligh and Dyer (1959), and cellular lipids using hexane:isopropanol (3:2, v/v), as detailed [16-18,20], and extracts dried under $\mathrm{N}_{2}$ before resuspension in isopropanol containing rat liver lipids, and separation by t.l.c. using petroleum ether: diethyl ether: glacial acetic acid (90:30:1 by vol.) as the mobile phase. Lipids were identified by comparison with authentic standards, and dpm determined by scintillation counting (Hidex 300SL); lipid mass was measured using colorimetric assays as described previously $[16-18,20]$.

\section{Gene and protein analyses}

Total RNA from liver $(60 \mathrm{mg})$ was isolated after homogenisation in $500 \mu \mathrm{l}$ of TriFast $^{\mathrm{Tw}}$ (PeqLab, UK); genomic DNA removed by treatment with DNase (Ambion', UK), and cDNA prepared by reverse transcription (Bioline, UK); negative controls, omitting reverse transcriptase enzyme, were included for each sample. Levels of mRNA encoding StarD proteins were measured relative to Gapdh, as described [16-18,20]; specific sequences for primers and fluorescent probes (FAM/TAMRA) are reported in Table 1. Gene and/or protein levels of StarD1 and StarD3 were reported [20].

Gene expression of Dgat1 and Dgat2, relative to Gapdh, were measured using commercial primers and Sybr Green (Maxima SYBR Green QPCR Master Mix), using the following primer sequences: Dgat1 sense (5'-CGGATAGCTTACAGTGTTCTG-3'), antisense (5'-CATCATACTCCATCATCTTCCTCA-3'); Dgat2 sense (5'-GCCAGGTGACAGAGAAGATG-3'), antisense (5'-GCAGCGAGAACAAGAATAAAG-3') and Gapdh, sense (5'-GTAACCAGGCGTCCGATAC-3') and antisense (5'-TCTCTGCTCCTCCCTGTTC-3'). Results are expressed as ratio to Gapdh, using the $2^{-\Delta \mathrm{Ct}}$ comparison method (Applied Biosystems)

Hepatic protein lysates were separated using 10\% (w/v) SDS PAGE gels, transferred to nitrocellulose membranes, and probed using rabbit polyclonal antibodies to StarD4 (1:1000), StarD5 (1:1000) and Gapdh $(1: 1000)$ as previously [16-18,20], except that fluorescently-labelled secondary antibodies (Licor) were employed and bands quantified using a Licor Odyssey FC and Image Studio software.

\section{Statistical analysis}

All values indicate mean \pm SEM; $n$ denotes numbers of independent determinations. Significant $(p<0.05)$ differences were determined by Student's t-test.

\section{Results}

\section{Hepatic expression of genes encoding the StarD family in $\mathrm{fa} /$ $f a$ and $F a / ?$ rats}

Gene expression of the StarD lipid trafficking proteins in liver samples from male and female, obese Zucker $(f a / f a)$ and lean $(F a / ?)$ rats (4 months old) are shown in Figure 1. Bioinformatic analyses of the $-3 \mathrm{~kb}$ promoter region of each family member revealed a number of putative response elements for lipid responsive transcription factors, including sterol element binding proteins and peroxisome proliferator 
Citation: Soffientini U, Dolan S, Graham A (2015) Cytosolic Lipid Trafficking Proteins STARD4 and STARD5 Modulate Hepatic Neutral Lipid Metabolism: Implications for Diabetic Dyslipidaemia and Steatosis. J Diabetes Metab 6: 558. doi:10.4172/2155-6156.1000558

Page 3 of 9

\begin{tabular}{|c|c|c|c|}
\hline Gene & $\begin{array}{l}\text { Forward primer sequence } \\
\qquad\left(5^{\prime}-3^{\prime}\right)\end{array}$ & $\begin{array}{l}\text { Reverse primer sequence } \\
\left(5^{\prime}-3^{\prime}\right)\end{array}$ & $\begin{array}{c}\text { Probe sequence } \\
\left(5^{\prime}-3^{\prime}\right)\end{array}$ \\
\hline StarD2 & GGACCCCCGCATCCAT & CGCTTTGAAGGTGGGAACA & TGCTACACCAACTGAAGCTCGCCCTT \\
\hline StarD4 & CAGAGTCTCCTGACAGGCTACATC & TGCTTGCCATTGCTGTGTCT & ACAGACCTGCGTGGCATGATCCC \\
\hline StarD5 & GCATCACGGATACGCTGTGT & CTGGGCGAATCAGCTTCAT & CAGAACСTCCACGСССТСАGСTG \\
\hline StarD6 & AAAAGAAGGTGCCATCTCAACAA & CAGATGAAGCCAAGGGTGTCT & TCTGGCTTCCACTGACCCAATGGC \\
\hline StarD7 & TGGAATGAGCAGTGAGGCTAA & GCCACAGCTACCCTCATTCTTC & CCACTGCTTCGTCCCCCGAGC \\
\hline StarD8 & CCTCACAAGCGAAGAGAATCTTG & GGCCTCCAGATGCAGTAGCA & AAGAAGATCGTTGCTGTCCACCTGACCA \\
\hline StarD9 & TTCAGTACCAGCCCCGTTGT & CGCAAGAAAACCAGGAATCC & CCGAACAAATCAACCСTCTCCGAGGTA \\
\hline StarD10 & TCCCCACGCTCTCTATGGAA & GACACCTTCACGGCCCAAAG & AGCCAGCTGCTTCAACAGAACCCCA \\
\hline StarD11 & AGACGAGTAGAGGAAGCATACAAGAAT & TCAAGAACTCTTCCTCATTAATCAGACT & AACCTCGCTTCGGAGGGCCAGACTAT \\
\hline StarD12 & GGGCAAACCAGACCAGAAG & TGCACTCGGCAATCATGTG & TCTGAATGAAAACCTAGCCGCCACTCA \\
\hline StarD13 & CAAGAAATCGAGGCGAAGGA & TGTTGATGGGAAACTGCGAAT & CTGCGAGCTGCCGGGTTCCC \\
\hline StarD14 & GCGCCGGCACATCAA & CTTCTGAGGCTGGTCATCTTTGT & AGCGCCTTCATGACTTTCGTGGTCC \\
\hline StarD15 & CGCCGGGCAACTACTTAAGT & CAGGAATCCCAGCATGCTT & ACTGCCTGCCTGGCCGCTGA \\
\hline Gapdh & CAACTACATGGTCTAGTTCCAGTATG & СTTCCCATTCTCAGCCTTGACGT & CTACCCACGGCAAGTTCAACGGCA \\
\hline
\end{tabular}

Table 1: Primer and probe sequences used to investigate gene expression of StarD proteins in samples of rodent liver.
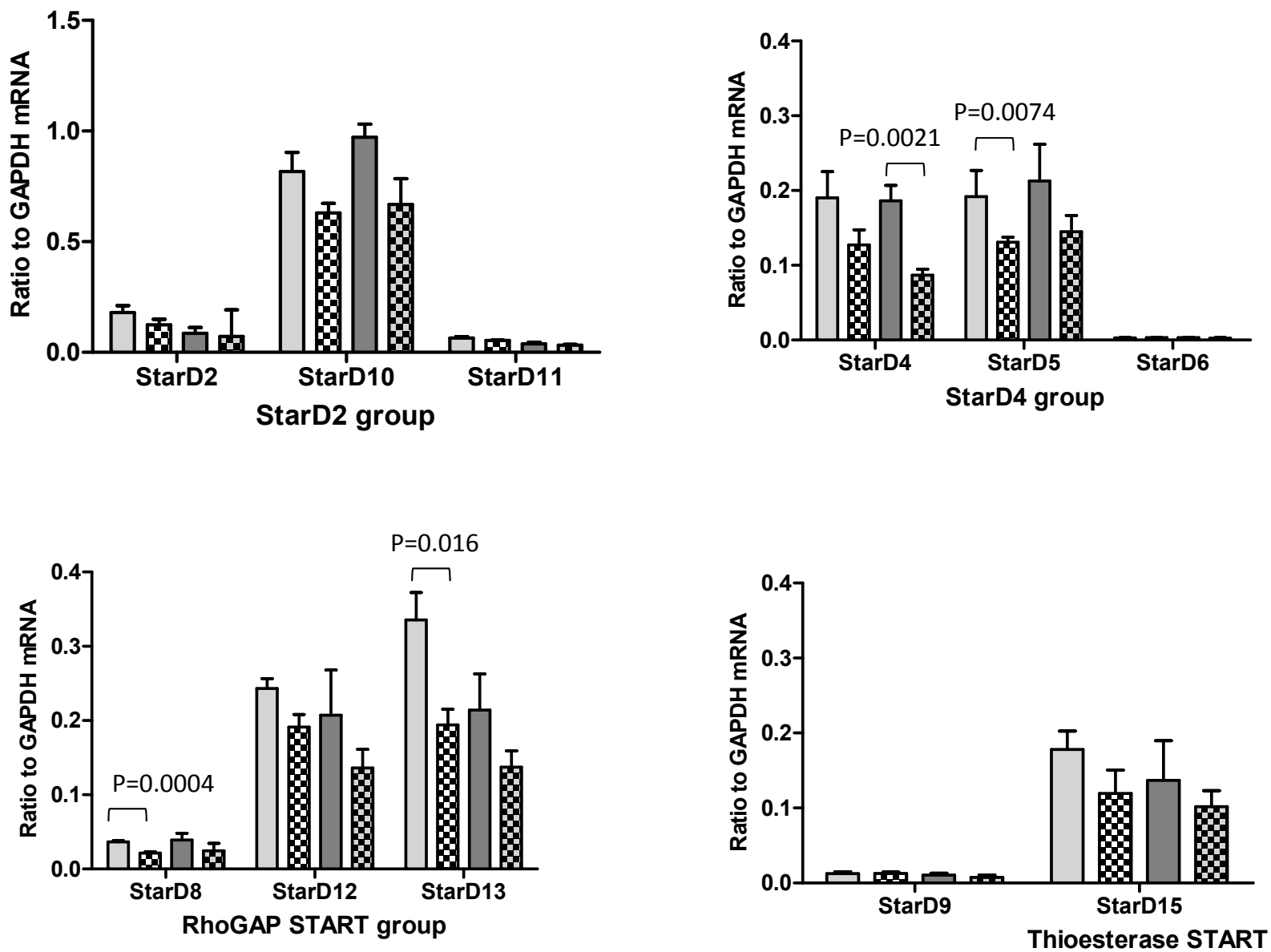

Figure 1: Gene expression, relative to Gapdh, of StarD2, StarD4, RhoGAP and Thioesterase START family subgroups, and StarD9, in lean (plain bars) and obese (hatched bars), male (light grey bars) and female (dark grey bars) Zucker rats; values are mean \pm SEM from four animals in each group.

activated receptors [data not shown]. Gene expression of 12 of the 15 mammalian START proteins were detected in rodent liver. Repression of StarD5 ( $p<0.01)$, StarD8 $(\mathrm{p}<0.01)$ and StarD13 $(p<0.05)$ were noted in obese male, compared with lean male rodents. Obesity in female rats was associated with decreased hepatic expression of Stard4 $(p<0.01)$ compared with lean female controls. The level of StarD6 mRNA was extremely low in hepatic tissues from male and female rodents.
No significant changes in expression were noted for genes encoding phospholipid and ceramide transporters (StarD2 subgroup), StarD9, RhoGAP StarD12, or the thioesterase, StarD15.

Hepatic expression of StarD4 and StarD5 proteins in $\mathrm{fa} / \mathrm{fa}$ and $F a /$ ? rats

Hepatic levels of StarD4 and StarD5 protein, expressed relative 
Citation: Soffientini U, Dolan S, Graham A (2015) Cytosolic Lipid Trafficking Proteins STARD4 and STARD5 Modulate Hepatic Neutral Lipid Metabolism: Implications for Diabetic Dyslipidaemia and Steatosis. J Diabetes Metab 6: 558. doi:10.4172/2155-6156.1000558

Page 4 of 9

to Gapdh, exhibited marked regulation compared with their gene expression (Figure 2). Obesity was associated with repression of hepatic StarD4 expression in both male $(15.6$-fold; $\mathrm{p}<0.01)$ and female $(6.55-$ fold; $\mathrm{p}<0.01)$ rats. By contrast, hepatic levels of StarD5 protein were significantly repressed $(3.2$-fold; $\mathrm{p}<0.001)$ by obesity in female, but not male rats, compared with lean controls.

\section{Cholesterol metabolism in hepatoma cells overexpressing STARD4 and STARD5}

Human cytosolic STARD4 and STARD5 proteins, and an empty vector (EV) control, were stably overexpressed in rat McArdle (McARH7777) hepatoma cells (Figure 3A); note that the data for the EV control was previously reported in [20]. Alignment of rodent and human amino acid sequences (ClustalW) revealed $84 \%$ and $88.78 \%$ identity, for StarD4/STARD4 and StarD5/STARD5, respectively. The efflux of $\left[{ }^{3} \mathrm{H}\right]$ cholesterol to exogenous human ApoA-I $\left(10 \mu \mathrm{g} \mathrm{ml}^{-1} ; 24 \mathrm{~h}\right)$ from STARD4 and STARD5 overexpressing cells are shown in Figure 3B. Overexpression of STARD4 significantly increased lipidation of ApoA-I by nearly two-fold, compared with EV and STARD5 overexpressing cells. The effects of overexpression of STARD4 and STARD5 on synthesis and secretion of de novo synthesized cholesterol and cholesteryl esters derived from $\left[{ }^{14} \mathrm{C}\right]$ acetate $(0.5 \mathrm{mM} ; 2 \mathrm{~h})$ are shown in Figures 4A to $4 \mathrm{D}$, and on the incorporation of $\left[{ }^{14} \mathrm{C}\right]$ oleate $(0.7 \mathrm{mM} ; 2 \mathrm{~h})$ into cellular and secreted cholesteryl esters, are shown in Figures $4 \mathrm{E}$ and $4 \mathrm{~F}$. The effect of STARD4 and STARD5, compared with the EV control, were compared under basal conditions, and after challenge with $0.35 \mathrm{mM}$ oleate to stimulate neutral lipid synthesis and secretion. A modest increase in secretion of $\left[{ }^{14} \mathrm{C}\right]$ cholesterol was noted under basal conditions ( $31 \%$; $\mathrm{p}<0.05)$ in STARD4 overexpressing cells. Measurement of incorporation of exogenous oleate into the cholesteryl ester pool (Figure 4E) revealed a significant decrease in STARD4 overexpressing cells, compared with STARD5 overexpressing cells; in the presence of $0.35 \mathrm{mM}$ cold oleate, this decline was also significant when compared with the EV control. No changes in secretion of cholesteryl $\left[{ }^{14} \mathrm{C}\right]$ oleate were evident (Figure $4 \mathrm{~F}$ ). Analysis of the total cholesterol mass of EV $\left(0.49 \pm 0.06 \mathrm{mg} \mathrm{mg}^{-1}\right.$ protein $)$, STARD4 $\left(0.46 \pm 0.16 \mathrm{mg} \mathrm{mg}^{-1}\right.$ protein) and STARD5 $\left(0.44 \pm 0.19 \mathrm{mg} \mathrm{mg}^{-1}\right.$ protein $)$ hepatocytes revealed no significant changes, either in the absence or presence of $0.35 \mathrm{mM}$ oleate.

Triacylglycerol synthesis in hepatoma cells overexpressing STARD4 and STARD5

The synthesis and secretion of triacylglycerol from $\left[{ }^{3} \mathrm{H}\right]$ glycerol

\section{$2 \mathrm{~A}$}

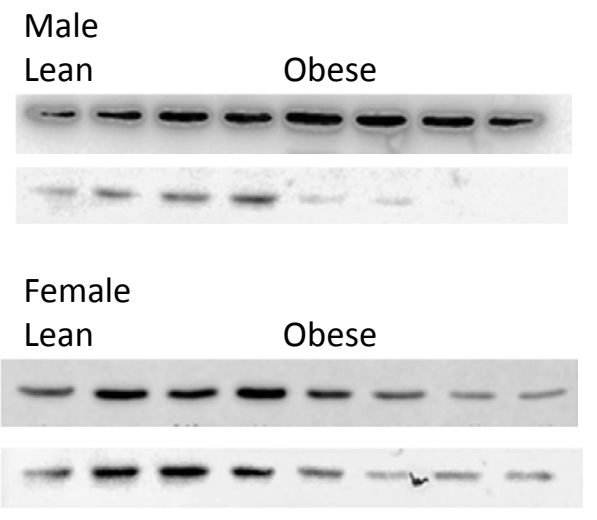

Gapdh 37kDa

StarD4 25kDa

Gapdh 37kDa

StarD4 25kDa

2B

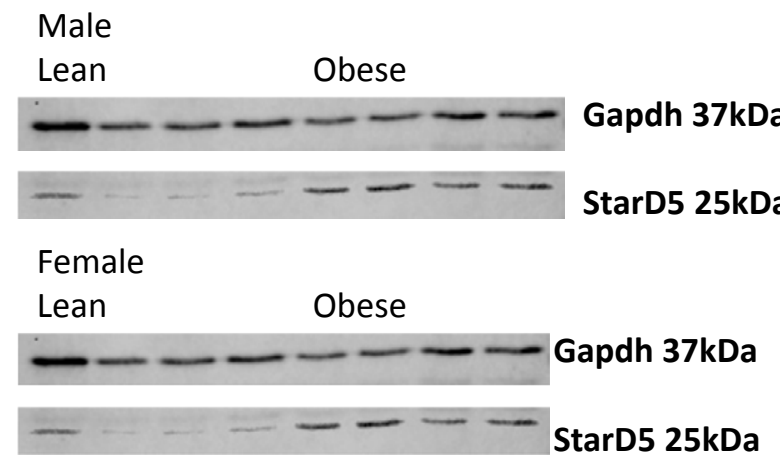

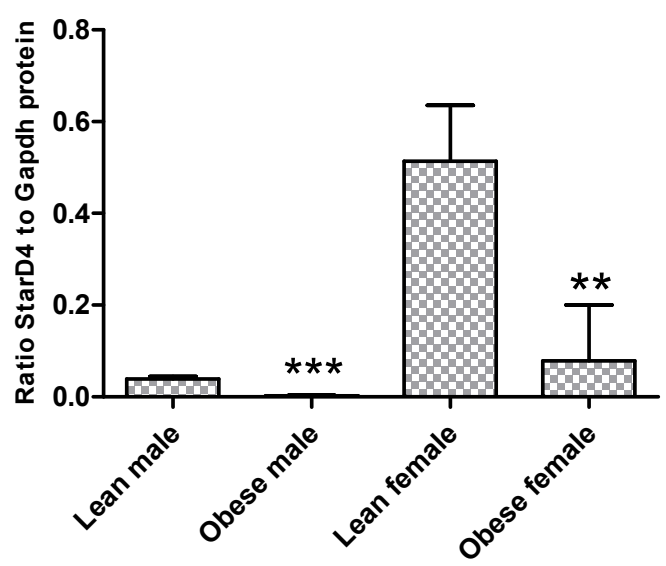

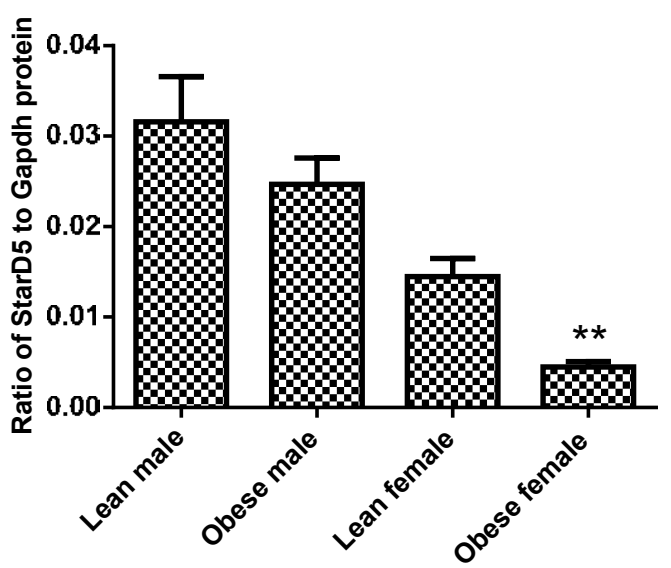

Figure 2: Expression of cytosolic StarD4 (2A) and StarD5 (2B) proteins, relative to Gapdh, in hepatic tissues from lean and obese, male and female Zucker rats; values are the mean \pm SEM from 4 animals in each group. 

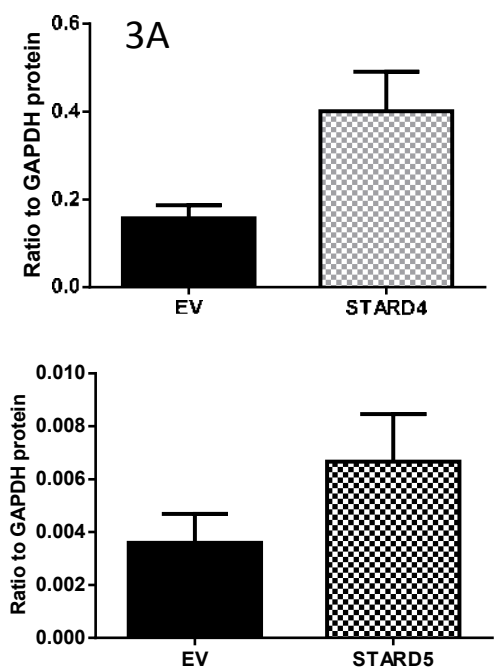

3B

EV STARD4

25kDa STARD4

37kDa Gapdh

EV STARD5

25kDa STARD5

37kDa Gapdh

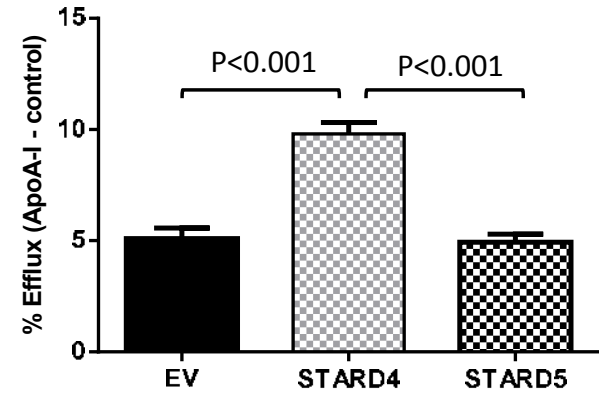

Figure 3: Expression of STARD4 and STARD5 in McArdle RH7777 hepatoma cells, stably transfected with empty vector (EV; pCMV.Neo), or the same vector

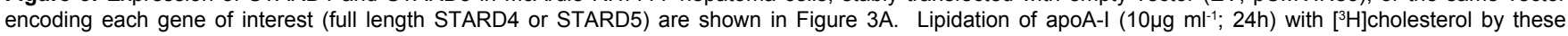
established cell lines is shown in Figure 3B. Values are mean \pm SEM for three independent experiments.
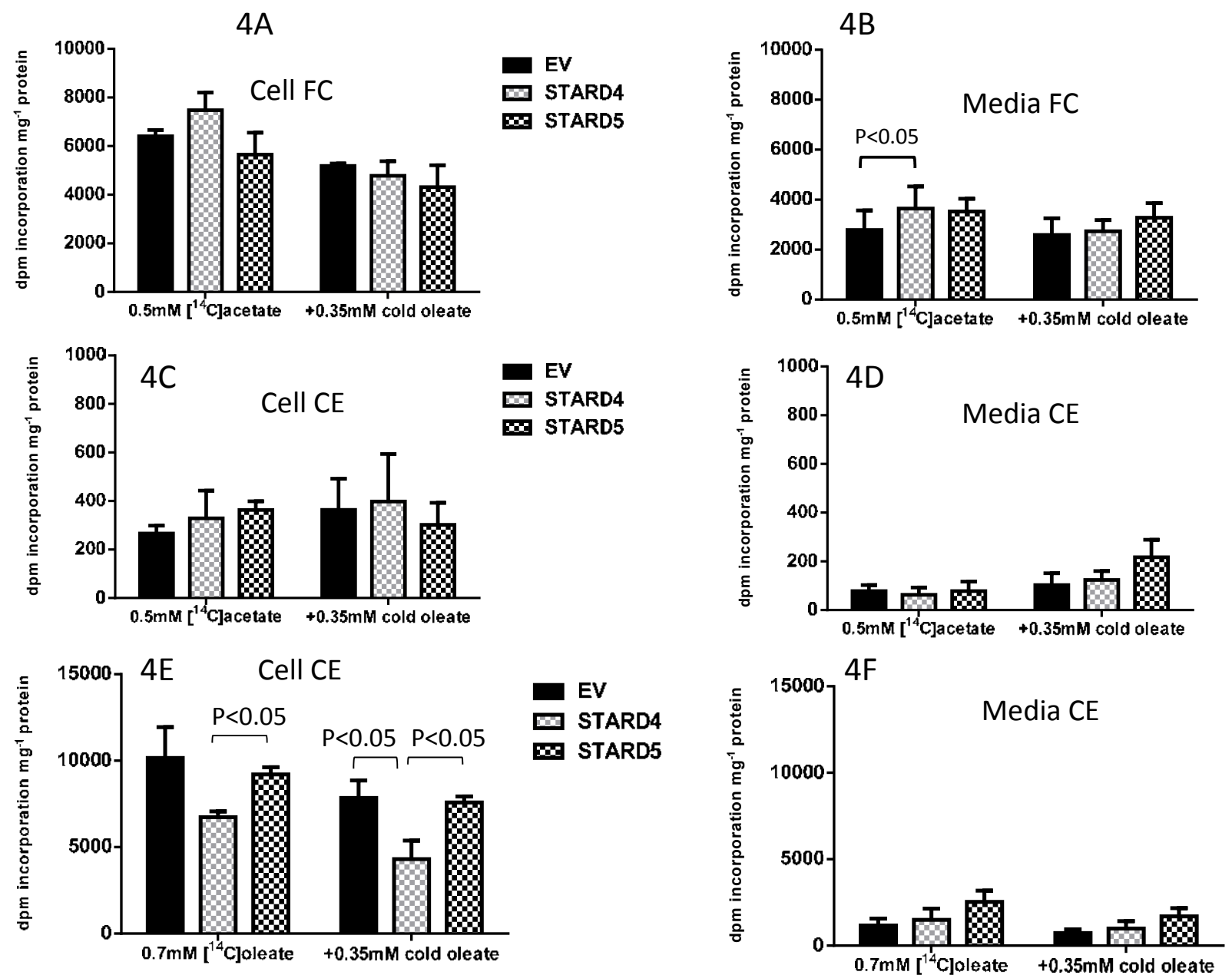

Figure 4: Incorporations $(2 \mathrm{~h})$ of $0.5 \mathrm{mM}\left[{ }^{14} \mathrm{C}\right]$ acetate into cellular $(4 \mathrm{~A})$ and secreted $(4 \mathrm{~B})$ cholesterol, cellular $(4 \mathrm{C})$ and secreted $(4 \mathrm{D})$ cholesteryl esters, and of $0.7 \mathrm{mM}\left[{ }^{14} \mathrm{C}\right]$ oleate into cellular (4E) and secreted (4F) cholesteryl ester pools, in the presence or absence of $0.35 \mathrm{mM}$ cold oleate, in EV, STARD4 and STARD5 overexpressing hepatoma cells. Values are mean \pm SEM for three independent experiments. 
Citation: Soffientini U, Dolan S, Graham A (2015) Cytosolic Lipid Trafficking Proteins STARD4 and STARD5 Modulate Hepatic Neutral Lipid Metabolism: Implications for Diabetic Dyslipidaemia and Steatosis. J Diabetes Metab 6: 558. doi:10.4172/2155-6156.1000558

Page 6 of 9

$(0.25 \mathrm{mM} ; 2 \mathrm{~h}),\left[{ }^{14} \mathrm{C}\right]$ acetate $(0.5 \mathrm{mM} ; 2 \mathrm{~h})$ and $\left[{ }^{14} \mathrm{C}\right]$ oleate $(0.7 \mathrm{mM}$; $2 \mathrm{~h})$ are shown in Figure 5. Again, the effect of STARD4 and STARD5, compared with EV control, were compared under basal conditions, and after challenge with $0.35 \mathrm{mM}$ oleate to stimulate neutral lipid synthesis and secretion. When total triacylglycerol synthesis and secretion was monitored using $\left[{ }^{3} \mathrm{H}\right]$ glycerol $(0.25 \mathrm{mM} ; 2 \mathrm{~h})$, the stimulatory effect of STARD5 overexpression on triacylglycerol synthesis was evident, at least under basal conditions (Figure 5A). No changes in secretion of triacyl $\left[{ }^{3} \mathrm{H}\right]$ glycerol were observed compared with the EV control (Figure 5B). No changes in the synthesis or secretion of triacylglycerol derived from endogenous lipogenesis were noted in cells overexpressing STARD4 or STARD5, compared with EV (Figures $5 \mathrm{C}$ and 5D, respectively). By contrast, overexpression of STARD5 stimulated the incorporation of exogenous $\left[{ }^{14} \mathrm{C}\right]$ oleate into the cellular (Figure 5E), but not secreted (Figure 5F), triacylglycerol pool, mirroring the findings in Figure 5A. The triacylglycerol mass tended to increase in the presence of $0.35 \mathrm{mM}$ oleate $(2 \mathrm{~h})$ in EV $\left(0.89 \pm 0.06 \mathrm{mg} \mathrm{mg}^{-1}\right.$ protein versus $0.35 \mathrm{mM}$ oleate $1.07 \pm 0.15 \mathrm{mg} \mathrm{mg}^{-1}$ protein; $\mathrm{n}=3$; non-significant (NS)), STARD4 (0.72 $\pm 0.26 \mathrm{mg} \mathrm{mg}^{-1}$ protein versus $1.01 \pm 0.23 \mathrm{mg} \mathrm{mg}^{-1}$ protein; $\mathrm{n}=3$; NS) and STARD5 overexpressing cells (0.75 $\pm 0.29 \mathrm{mg}$ $\mathrm{mg}^{-1}$ protein versus $1.32 \pm 0.47 \mathrm{mg} \mathrm{mg}^{-1}$ protein; $\mathrm{n}=3$; NS).

The expression levels of Dgat1 and Dgat2 in EV, STARD4 and STARD5 overexpressing hepatoma cell lines are shown in Figure 6. Overexpression of STARD4 increased expression of Dgat1, but decreased the levels of Dgat2 mRNA levels, compared with the EV control. By contrast, overexpression of STARD5 enhanced expression of both Dgat1 and Dgat2.

\section{Discussion}

Obesity alters gene expression of StarD4 and Rho-GAP subgroups of the START family of lipid trafficking proteins, which may impact on the efficiency of hepatic lipid transport, a key issue in diabetic dyslipidaemia and non-alcoholic fatty liver disease (Figure 1). At four months, $f a / f a$ rats are normoglycaemic and hyperinsulinaemic [14], hyperlipidaemic and steatotic, compared with lean controls [20]; moreover, obese male Zucker rats exhibit greater accumulation of hepatic triacylglycerol and phospholipid mass than obese female rats,
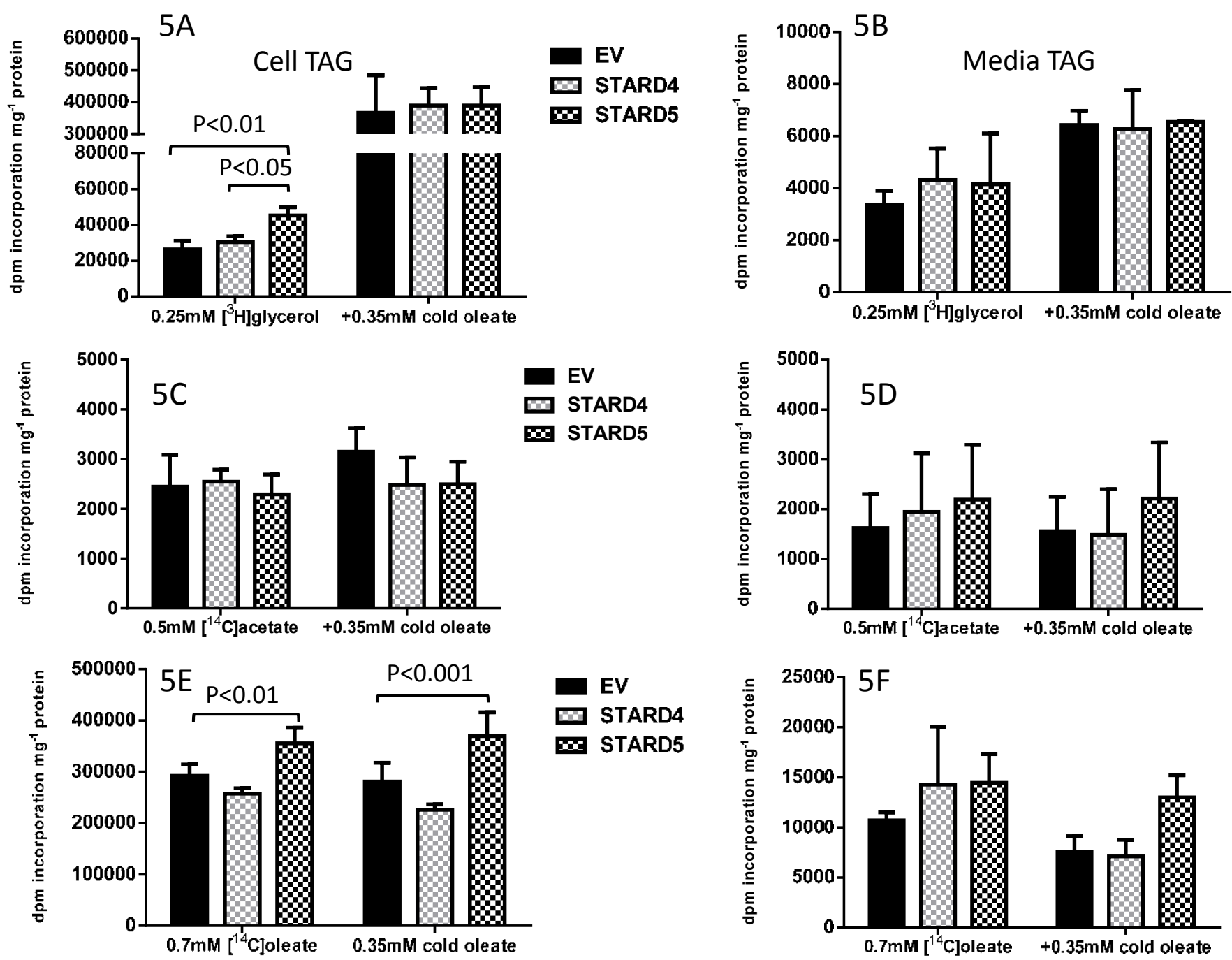

Figure 5: Incorporations $(2 \mathrm{~h})$ of $0.25 \mathrm{mM}\left[{ }^{3} \mathrm{H}\right]$ glycerol, $0.5 \mathrm{mM}\left[{ }^{14} \mathrm{C}\right]$ acetate and $0.7 \mathrm{mM}\left[{ }^{14} \mathrm{C}\right]$ oleate into cellular $(5 \mathrm{~A}, 5 \mathrm{C}, 5 \mathrm{E})$ and secreted $(5 \mathrm{~B}, 5 \mathrm{D}, 5 \mathrm{~F})$ triacylglycerol, in the presence and absence of $0.35 \mathrm{mM}$ cold oleate, in EV, STARD4 and STARD5 overexpressing hepatoma cells. Values are mean \pm SEM for three independent experiments. 
Citation: Soffientini U, Dolan S, Graham A (2015) Cytosolic Lipid Trafficking Proteins STARD4 and STARD5 Modulate Hepatic Neutral Lipid Metabolism: Implications for Diabetic Dyslipidaemia and Steatosis. J Diabetes Metab 6: 558. doi:10.4172/2155-6156.1000558

Page 7 of 9
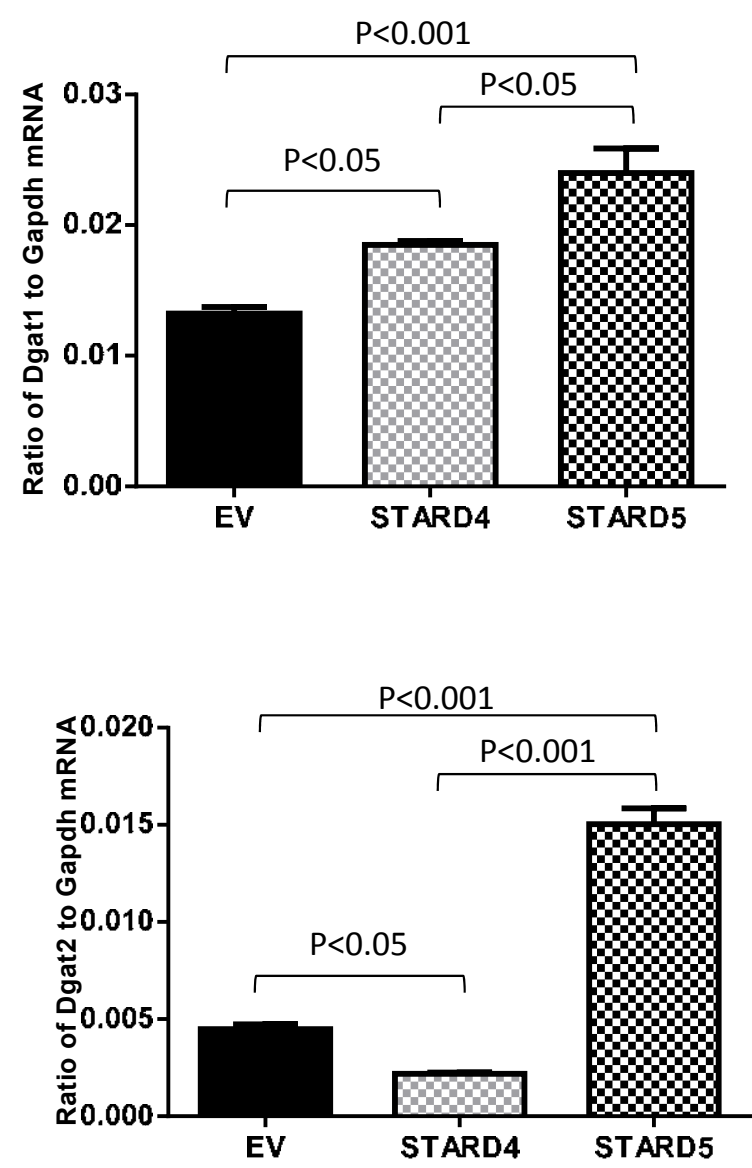

Figure 6: The expression of Dgat1 and Dgat2, relative to Gapdh, in EV, STARD4 and STARD5 overexpressing McA-RH7777 hepatoma cells; values are the mean \pm SEM of quadruplicate determinations by Q-PCR.

while a more profound hyperlipidaemia is evident in female rats [20,21]. Interpreting the outcome of individual changes in gene expression is complex, as the relationship between StarD mRNA and protein levels is not necessarily linear, presumably mediated via post-translational mechanisms (Figure 2). However, it is tempting to speculate that repression of genes encoding tumour suppressor proteins, StarD8 and StarD13, could contribute to the incidence of hepatocellular carcinoma associated with obesity [22].

Clearly, however, hepatic expression of StarD4 protein, relative to Gapdh, is repressed by obesity in male and female rats. Levels of StarD4 mRNA and protein have previously been shown to be repressed by cholesterol accumulation in fibroblasts and liver tissues [23,24], while female mice are more affected by global genetic deletion of StarD4 than male animals [11]. By contrast, StarD5 is expressed at much lower levels than StarD4 in liver tissues, when expressed as a ratio to Gapdh, and is repressed by obesity only in female rats. Previous work has suggested that StarD5 is found within the sinusoidal lining cells in the human liver, co-localized with CD68, a marker for Kupffer cells [25]; however, StarD5 protein was clearly present in McArdle hepatoma cells (Figure 3). Unlike StarD4, which is regulated by sterol regulatory element binding protein -2 (SREBP-2) and is therefore repressed in tissues accumulating cholesterol [23,24], the promoter region of StarD5 does not contain a SRE [24]. Instead, StarD5 expression increases in macrophages loaded with free cholesterol [24], as a consequence of activation of the endoplasmic reticulum stress response. These findings led to the suggestion that StarD4 and StarD5 might serve differing roles in lipid metabolism [24].

Indeed, given the hepatic and serum lipid profile of $f a / f a$ rats [20], we initially speculated that StarD4 might facilitate lipid export from the liver, while StarD5 could be linked with hepatic lipid accumulation. Certainly, overexpression of STARD4 increased the lipidation of exogenous apoA-I, one measure of hepatic ABCA1 activity [21] suggesting an association with serum or HDL lipid levels (Figure 3B). A small increase in secretion of endogenously synthesized cholesterol was also noted in the absence of apoA-I, which may reflect efflux to endogenously produced apoA-I. However, despite the reciprocal relationship that exists between hepatic ABCA1 activity and output of VLDL [26-28], and evident changes in Dgat1/Dgat2 ratio (Figure 6), no changes in synthesis or secretion of triacylglycerol, from either endogenous or exogenous fatty acids, were observed in STARD4 overexpressing cells. Instead, reduced incorporation of exogenous $\left[{ }^{14} \mathrm{C}\right]$ oleate into the cholesteryl ester pool was evident in STARD4 overexpressing hepatoma cells, compared with STARD5 overexpressing cells or EV controls (Figure 4E). This was particularly surprising, given previous findings indicating that StarD4/STARD4 activity is associated with increased delivery of sterol to the endocytic recycling compartment (ERC) and the endoplasmic reticulum and enhances ACAT-dependent deposition of cholesterol ester mass in macrophages, fibroblasts, hepatocytes and hepatoma cells [29-32].

The most obvious difference in our study, compared with these reports [29-32], is the challenge posed by exposure to a physiologically relevant $(0.7 \mathrm{mM})$ concentration (of (radiolabelled) oleate in the culture medium. It is possible that STARD4 can act as a bi-directional cholesterol transporter, capable of shuttling cholesterol to and from the endoplasmic reticulum, depending upon the cellular milieu. Certainly, overexpression of STARD4 facilitated removal of cholesterol from the plasma membrane (Figure 3B). In good agreement with the former, knockdown of STARD4 led to retention of cholesterol at the plasma membrane in HepG2 cells [32]. STARD4 can also facilitate bile acid formation [29], so that cholesterol may be preferentially directed towards metabolism rather than storage: the McArdle RH7777 cell line used in this study expresses both cholesterol 7a-hydroxylase and CYP27A1 [20].

Neutral lipid metabolism was differentially regulated by STARD5, compared with STARD4, in McArdle hepatoma cells. No observable effects on cholesterol efflux, endogenous cholesterol and cholesteryl ester biosynthesis, and incorporation of exogenous oleate into the cholesteryl ester pool were evident. Indeed, controversy exists as to whether StarD5 actually binds sterols [33-36]. Rodriguez-Agudo et al. [33] reported that StarD5 bound cholesterol and 25-hydroxycholesterol, and that overexpression of this protein in hepatocytes increased the free cholesterol content of intracellular membranes [33]. By contrast, Letourneau et al. [34-36] utilised nuclear magnetic resonance, circular dichroism and isothermal titration calorimetry to demonstrate that primary and secondary bile acids, and not cholesterol, were the ligands for StarD5, suggesting that this protein is involved in the cellular responses elicited by bile acids.

In our study, overexpression of STARD5 promotes the total synthesis of triacylglycerol under basal conditions (Figure 5A) and the incorporation of exogenous $\left[{ }^{14} \mathrm{C}\right]$ oleate into this pool, in the presence or absence of cold oleate (Figure 5E), possibly reflecting the increased expression of Dgat1 (Figure 6). No changes in endogenous lipogenesis from $\left[{ }^{14} \mathrm{C}\right]$ acetate were noted in STARD5 overexpressing cells, compared with the EV control, despite the fact that Dgat2 expression 
Citation: Soffientini U, Dolan S, Graham A (2015) Cytosolic Lipid Trafficking Proteins STARD4 and STARD5 Modulate Hepatic Neutral Lipid Metabolism: Implications for Diabetic Dyslipidaemia and Steatosis. J Diabetes Metab 6: 558. doi:10.4172/2155-6156.1000558

Page 8 of 9

levels were also increased (Figure 6). Overexpression of STARD5 may therefore preferentially regulate diacylglycerol transferase-1 (DGAT1) activity rather than DGAT-2. DGAT-1 has dual topology, with cytosolic (overt) and lumenal (latent) activities which, respectively, allow remodelling of the triacylglycerol pool within the cytosol, and the generation of triacylglycerol used to lipidate nascent VLDL prior to secretion [37]. The activity of diacylglycerol acyl transferase-2 (DGAT2) is restricted to the cytosolic aspect of the endoplasmic reticulum. This enzyme utilises glycerol-3-phosphate and de novo synthesized fatty acids as substrates to form de novo synthesized triacyglycerol [reviewed in 37]. A minor fraction of this triacylglycerol is utilised for secretion with apoB, and some is stored as cytosolic lipid droplets; however, the majority is hydrolysed to yield partial glycerides which are then re-esterified by DGAT-1, which utilises exogenously derived fatty acids. The outcomes here suggest enhancement of overt DGAT-1 activity in STARD5 overexpressing cells, although the mechanism(s) by which this is achieved remain to be established.

Finally, our study suggests that variations in hepatic levels of STARD4 or STARD5 proteins may contribute to the altered lipid metabolism that may predispose individuals to future development of diabetes. It is possible that familial inheritance of altered expression levels of STARD4 or STARD5 may contribute to the increased risk of coronary heart disease observed even in non-diabetic subjects [reviewed in 38]. Certainly, increased circulating levels of non-esterified fatty acids and triglycerides can lead to ectopic lipid accumulation and predicate cardiac dysfunction in 'pre-diabetic' and diabetic individuals [38].

\section{Conclusions}

The cytosolic lipid trafficking proteins, STARD4 and STARD5, appear to exert differing effects on neutral lipid metabolism in McArdle RH-7777 hepatoma cells. Cytosolic STARD4 impacts predominantly on cholesterol efflux to apoA-I, implying directional transport of cholesterol to the plasma membrane, and limiting the incorporation of radiolabelled oleate into the hepatic cholesteryl ester pool. By contrast, STARD5 does not alter hepatic cholesterol metabolism, but increases the basal synthesis of triacylglycerol and enriches the hepatic triacylglycerol pool with exogenous (preformed) fatty acids, possibly playing a permissive role in hepatic steatosis. Thus, altered levels of cytosolic StarD proteins may be implicated in the pathogenesis associated with dyslipidaemia and/or steatosis. Further studies in murine models of obesity and diabetes are clearly warranted, while investigation of the levels of StarD proteins in diabetic patients could provide new insights regarding their role(s) as markers of predisposition to cardiovascular disease and diabetes.

\section{Acknowledgements}

The authors would like to thank the excellent technical team in the Department of Life Sciences, GCU. The authors are indebted to GCU for the studentship supporting US, and to Diabetes UK for a small grant (11/0004333).

\section{References}

1. Olkkonen VM, Li S (2013) Oxysterol-binding proteins: sterol and phosphoinositide sensors coordinating transport, signaling and metabolism. Prog Lipid Res 52: 529-538.

2. Weber-Boyvat M, Zhong W, Yan D, Olkkonen VM (2013) Oxysterol-binding proteins: functions in cell regulation beyond lipid metabolism. Biochem Pharmacol 86: 89-95

3. Soccio RE, Breslow JL (2003) StAR-related lipid transfer (START) proteins: mediators of intracellular lipid metabolism. J Biol Chem 278: 22183-22186.

4. Clark BJ (2012) The mammalian START domain protein family in lipid transport in health and disease. J Endocrinol 212: 257-275.
5. Alpy F, Tomasetto C (2014) START ships lipids across interorganelle space. Biochimie 96: 85-95

6. Lavigne P, Najmanivic R, Lehoux JG (2010) Mammalian StAR-related lipid transfer (START) domains with specificity for cholesterol: structural conservation and mechanism of reversible binding. Subcell Biochem 51: 425437

7. Bose HS, Sugawara T, Strauss JF 3rd, Miller WL; International Congenital Lipoid Adrenal Hyperplasia Consortium (1996) The pathophysiology and genetics of congenital lipoid adrenal hyperplasia. N Engl J Med 335: 18701878.

8. Wang X, Rao TP, Kosakowska-Cholody T, Masood MA, Southon E, et al (2009) Mitochondrial degeneration and not apoptosis is the primary cause of embryonic lethality in ceramide transfer protein mutant mice. J Cell Biol 184 143-158.

9. Durkin ME, Avner MR, Huh CG, Yuan BZ, Thorgeirsson SS, et al. (2005) DLC1 , a Rho GTPase-activating protein with tumor suppressor function, is essential for embryonic development. FEBS Lett 579: 1191-1196.

10. Kishida T, Kostetskii I, Zhang Z, Martinez F, Liu P, et al. (2004) Strauss JF 3rd: Targeted mutation of the MLN64 START domain causes only modest alterations in cellular sterol metabolism. J Biol Chem 279: 19276-19285.

11. Riegelhaupt JJ, Waase MP, Garbarino J, Cruz DE, Breslow JL (2010) Targeted disruption of steroidogenic acute regulatory protein D4 leads to modest weight reduction and minor alterations in lipid metabolism. J Lipid Res 51: 1134-1143.

12. Scapa EF, Pocai A, Wu MK, Gutierrez-Juarez R, Glenz L, et al. (2008) Regulation of energy substrate utilisation and hepatic insulin sensitivity by phosphatidylcholine transfer protein/StarD2. FASEB J 22: 2579-2590.

13. Lin Y, Chen NT, Shih YP, Liao YC, Xue L, et al. (2010) DLC2 modulates angiogenic responses in vascular endothelial cells by regulating cell attachment and migration. Oncogene 29: 3010-3016.

14. Iannitti T, Graham A, Dolan S (2012) Increased central and periphera inflammation and inflammatory hyperalgesia in Zucker rat model of leptin receptor deficiency and genetic obesity. Exp Physiol 97: 1236-1245.

15. Bligh EG, Dyer WJ (1959) A rapid method of total lipid extraction and purification. Can J Biochem Physiol 37: 911-917.

16. Borthwick F, Taylor JM, Bartholomew C, Graham A (2009) Differential regulation of the STARD1 subfamily of START lipid trafficking proteins in human macrophages. FEBS Lett 583: 1147-1153.

17. Taylor JM, Borthwick F, Bartholomew C, Graham A (2010) Overexpression of steroidogenic acute regulatory protein increases macrophage cholesterol efflux to apolipoprotein Al. Cardiovasc Res 86: 526-534.

18. Borthwick F, Allen AM, Taylor JM, Graham A (2010) Overexpression of STARD3 in human monocyte/macrophages induces an anti-atherogenic lipid phenotype. Clin Sci (Lond) 119: 265-272.

19. Hansson PK, Asztely AK, Clapham JC, Schreyer SA (2004) Glucose and fatty acid metabolism in McA-RH7777 hepatoma cells vs. rat primary hepatocytes: responsiveness to nutrient availability. Biochim Biophys Acta 1684: 54-62.

20. Soffientini U, Caridis AM, Dolan S, Graham A (2014) Intracellular cholestero transporters and modulation of hepatic lipid metabolism: implications for diabetic dyslipidaemia and steatosis. Biochim Biophys Acta Molecular and Cell Biology of Lipids 1841: 1372-1382.

21. Basso F, Freeman L, Knapper CL, Remaley A, Stonik J, et al. (2003) Role of the hepatic ABCA1 transporter in modulating intrahepatic cholesterol and plasma HDL cholesterol concentrations. J Lipid Res 44: 296-302.

22. Caldwell SH, Crespo DM, Kang HS, Al-Osaimi AM (2004) Obesity and hepatocellular carcinoma. Gastroenterology 127: S97-103.

23. Soccio RE, Adams RM, Romanowski MJ, Sehayek E, Burley SK, et al. (2002) The cholesterol-regulated StarD4 gene encodes a StAR-related lipid transfer protein with two closely related homologues, StarD5 and StarD6. Proc Nat Acad Sci U S A 99: 6943-6948.

24. Soccio RE, Adams RM, Maxwell KN, Breslow JL (2005) Differential gene regulation of StarD4 and StarD5 cholesterol transfer proteins. Activation of StarD4 by sterol regulatory element-binding protein- 2 and StarD5 by endoplasmic reticulum stress. J Biol Chem 280: 19410-19418.

25. Rodriguez-Agudo D, Ren S, Hylemon PB, Montañez R, Redford K, et al. (2006) 
Citation: Soffientini U, Dolan S, Graham A (2015) Cytosolic Lipid Trafficking Proteins STARD4 and STARD5 Modulate Hepatic Neutral Lipid Metabolism: Implications for Diabetic Dyslipidaemia and Steatosis. J Diabetes Metab 6: 558. doi:10.4172/2155-6156.1000558

Page 9 of 9

Localization of StarD5 cholesterol binding protein. J Lipid Res 47: 1168-1175.

26. Chung S, Gebre AK, Seo J, Shelness GS, Parks JS (2010) A novel role for ABCA1-generated large pre-beta migrating nascent $\mathrm{HDL}$ in the regulation of hepatic VLDL triglyceride secretion. J Lipid Res 51: 729-742.

27. Chung S, Timmins JM, Duong M, Degirolamo C, Rong S, et al. (2010) Targeted deletion of hepatocyte ABCA1 leads to very low density lipoprotein triglyceride overproduction and low density lipoprotein hypercatabolism. J Biol Chem 285 12197-12209.

28. Liu M, Chung S, Shelness GS, Parks JS (2012) Hepatic ABCA1 and VLDL triglyceride production. Biochim Biophys Acta 1821: 770-777.

29. Rodriguez-Agudo D1, Ren S, Wong E, Marques D, Redford K, et al. (2008) Intracellular cholesterol transporter StarD4 binds free cholesterol and increases cholesteryl ester formation. J Lipid Res 49: 1409-1419.

30. Rodriguez-Agudo D1, Calderon-Dominguez M, Ren S, Marques D, Redford $\mathrm{K}$, et al. (2011) Subcellular localization and regulation of StarD4 protein in macrophages and fibroblasts. Biochim Biophys Acta 1811: 597-606.

31. Mesmin B, Pipalia NH, Lund FW, Ramlall TF, Sokolov A, et al. (2011) STARD4 abundance regulates sterol transport and sensing. Mol Biol Cell 22: 4004-4015.

32. Garbarino J, Pan M, Chin HF, Lund FW, Maxfield FR, et al. (2012) STARD4 knockdown in HepG2 cells disrupts cholesterol trafficking associated with the plasma membrane, ER, and ERC. J Lipid Res 53: 2716-2725.
33. Rodriguez-Agudo D, Ren S, Hylemon PB, Redford K, Natarajan R, et al. (2005) Human StarD5, a cytosolic StAR-related lipid binding protein. J Lipid Res 46 1615-1623.

34. Létourneau D, Lorin A, Lefebvre A, Frappier V, Gaudreault F, et al. (2012) StAR-related lipid transfer domain protein 5 binds primary bile acids. J Lipid Res 53: 2677-2689.

35. Létourneau D, Lefebvre A, Lavigne P, LeHoux JG (2013) STARD5 specific ligand binding: comparison with STARD1 and STARD4 subfamilies. Mol Cell Endocrinol 371: 20-25

36. Létourneau D, Lorin A, Lefebvre A, Cabana J, Lavigne $P$, et al. (2013) Thermodynamic and solution state NMR characterization of the binding of secondary and conjugated bile acids to STARD5. Biochim Biophys Acta 1831 1589-1599.

37. Zammit VA (2013) Hepatic triacylglycerol synthesis and secretion: DGAT2 as the link between glycaemia and triglyceridaemia. Biochem J 451: 1-12.

38. Ciccone MM, Scicchitano P, Cameli M, Cecere A, Cortese F, et al. (2014) Endothelial function in pre-diabetes, diabetes and diabetic cardiomyopathy: $A$ Review. J Diabetes Metab 5: 364.
Citation: Soffientini U, Dolan S, Graham A (2015) Cytosolic Lipid Trafficking Proteins STARD4 and STARD5 Modulate Hepatic Neutral Lipid Metabolism: Implications for Diabetic Dyslipidaemia and Steatosis. J Diabetes Metab 6: 558. doi:10.4172/2155-6156.1000558
Submit your next manuscript and get advantages of OMICS Group submissions

Unique features:

- User friendly/feasible website-translation of your paper to 50 world's leading languages

- Audio Version of published paper

Digital articles to share and explore

Special features:

400 Open Access Journals

30,000 editorial team

21 days rapid review process

Quality and quick editorial, review and publication processing

- Indexing at PubMed (partial), Scopus, EBSCO, Index Copernicus and Google Scholar etc

- Sharing Option: Social Networking Enabled

- Authors, Reviewers and Editors rewarded with online Scientific Credits

- Better discount for your subsequent articles

Submit your manuscript at: www.editorialmanager.com/acrgroup 